\title{
ORTHOMADS: A deterministic MADS instance with orthogonal directions *
}

\author{
Mark A. Abramson ${ }^{\dagger} \quad$ Charles Audet $\ddagger \quad$ J.E. Dennis Jr. $\S$ \\ Sébastien Le Digabel \\ 28th February 2008
}

\begin{abstract}
The purpose of this paper is to introduce a new way of choosing directions for the Mesh Adaptive Direct Search (MADS) class of algorithms. The advantages of this new ORTHOMADS instantiation of MADS are that the polling directions are chosen deterministically, ensuring that the results of a given run are repeatable, and that they are orthogonal to each other, therefore the convex cones of missed directions at each iteration are minimal in size.

The convergence results for ORTHOMADS follow directly from those already published for MADS, and they hold deterministically, rather than with probability one, as for LTMADS, the first MADS instance. The initial numerical results are quite good for both smooth and nonsmooth, and constrained and unconstrained problems considered here.
\end{abstract}

Keywords: Mesh Adaptive Direct Search algorithms (MADS), deterministic, orthogonal directions, constrained optimization, nonlinear programming.

*Work of the first author was supported by FCAR grant NC72792 and NSERC grant 239436-05. The third author was supported by LANL 94895-001-04 34, and both were supported by AFOSR FA9550-071-0302, the Boeing Company, ExxonMobil Upstream Research Company.

${ }^{\dagger}$ Air Force Institute of Technology, Department of Mathematics and Statistics, 2950 Hobson Way, Bldg 641, Wright Patterson AFB, Ohio 45433 USA, www.afit.edu/en/ENC/Faculty/MAbramson/abramson.html, Mark.Abramson@afit.edu.

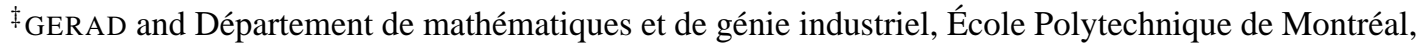
C.P. 6079, Succ. Centre-ville, Montréal, Québec H3C 3A7 Canada, www.gerad.ca/Charles.Audet, Charles.Audet@gerad.ca.

$\S$ Computational and Applied Mathematics Department, Rice University - MS 134, 6100 South Main Street, Houston, Texas 77005-1892 USA, www.caam.rice.edu/ dennis, dennis@ rice.edu.

`GERAD and Département de mathématiques et de génie industriel, Ecole Polytechnique de Montréal, C.P. 6079, Succ. Centre-ville, Montréal, Québec H3C 3A7 Canada, Sebastien.Le.Digabel@gerad.ca. 


\section{Introduction}

This paper considers optimization problems of the form

$$
\min _{x \in \Omega} f(x),
$$

where $f: \Omega \subset \mathbb{R}^{n} \rightarrow \mathbb{R} \cup\{\infty\}$ is typically evaluated through a black-box computer simulation with no available derivatives, and $\Omega$ is a set of feasible points also defined by black-box nonlinear constraint, or even Boolean, functions. Because no exploitable information on the nature of $f$ or $\Omega$ exists, we consider direct search methods which only use functions evaluations to drive their search.

Mesh Adaptive Direct Search (MADS) is introduced in [4] as a SEARCH/POLL derivative-free class of methods with strong convergence properties. It extends the Generalized Pattern Search (GPS) method of [18]. The constraints are treated by the extreme barrier approach, which simply rejects points outside $\Omega$ by setting their objective function value to $\infty$. The first instance of this class of methods is called LTMADS.

LTMADS behaves well in practice, but it has drawbacks that we wish to correct in this paper. First, there is a probabilistic component to the choice of polling directions. For each new mesh size, a random direction is chosen to a current mesh point. That direction is completed somewhat randomly to a positive spanning set of directions from the current iterate to other current mesh points. The resulting algorithm is shown to have Clarke stationary point convergence with probability one. However, it has been observed [10] that this way of choosing polling directions can lead to undesirably large angles between some of the members of the LTMADS polling set at a given iteration.

The purpose of this paper is to introduce a new variant of MADS, which we call ORTHOMADS, that uses an orthogonal positive spanning set of polling directions and thus avoids large angles between polling directions. In Figure 3, we show some experiments in which the ORTHOMADS directions do seem better distributed than the LTMADS.

We show that ORTHOMADS shares the same theoretical convergence results as LTMADS, except that the convergence is not qualified by being of probability one. In the tests given here, ORTHOMADS performs generally better than LTMADS.

ORTHOMADS is detailed in Section 2, where we show a deterministic way to construct a polling set on the current mesh of orthogonal polling directions (the ORTHOMADS directions). Section 2 also gives the convergence results, based on those in [4]. Finally, we present numerical results in Section 3 and some concluding remarks in Section 4.

Notation: Throughout the text, $\|\cdot\|$ denotes the $\ell_{2}$ norm, $e_{i} \in \mathbb{R}^{n}$ is the $i^{\text {th }}$ coordinate vector, and $e \in \mathbb{R}^{n}$ is the vector whose components are all equal to 1 . $B_{\varepsilon}(x)$ denotes the open ball of radius $\varepsilon$ around $x$. 


\section{The ORTHOMADS algorithm}

The ORTHOMADS algorithm is described in this section. We will not give details for the MADS class of algorithms and its LTMADS instantiation, since they are available in [4].

Each MADS iteration $k$ is separated into two steps, the SEARCH and the POLL, where the objective function $f$ and the test for feasibility are evaluated at finitely many trial points. These trial points lie on the mesh $M_{k}$ defined by

$$
M_{k}=\left\{x+\Delta_{k}^{m} D z: x \in V_{k}, z \in \mathbb{N}^{n_{D}}\right\} \subset \mathbb{R}^{n},
$$

where $V_{k} \subset \mathbb{R}^{n}$ is the set of all evaluated points by the start of the iteration, $\Delta_{k}^{m} \in \mathbb{R}_{+}$ is the mesh size parameter at iteration $k$, and $D$ is a matrix in $\mathbb{R}^{n \times n_{D}}$ composed of $n_{D}$ directions in $\mathbb{R}^{n}$. This paper focuses on the POLL step which is characterized by the set of trial points

$$
P_{k}=\left\{x_{k}+\Delta_{k}^{m} d: d \in D_{k}\right\} \subset M_{k},
$$

where $x_{k}$ is the POLL center at iteration $k$ and $D_{k}$ is the set of POLL directions, which have to form a positive spanning set and to be constructed so that POLL trial points lie on the mesh $M_{k}$. In GPS, a related method, the directions contained in $D_{k}$ are always chosen among the columns of $D$. Therefore, in GPS, there is only the same finite number of possibilities for selecting the directions in every $D_{k}$.

The differences between LTMADS and ORTHOMADS lie in the way to generate the directions in $D_{k}$ : With LTMADS, $D_{k}$ is randomly generated and directions are not necessarily orthogonal, possibly leading to large angles between directions and large unexplored convex cones of directions at a given step. However, the union of all normalized LTMADS directions over all iterations $k$ is dense in the unit sphere with probability one.

ORTHOMADS introduces a new way to generate the POLL directions $D_{k}$. This new method is deterministic and generates orthogonal directions, which together with their negatives form $D_{k}$, and such that the union of all normalized ORTHOMADS directions over all iterations is dense in the unit sphere. Furthermore, the components of these directions are integer, so that POLL points lie on the mesh defined with $D=\left[I_{n}-I_{n}\right]$, where $I_{n}$ is the identity matrix in dimension $n$. The orthogonality of the ORTHOMADS directions offers a better distribution of the POLL trial points in the search space, and the advantage of determinism is that numerical results are now easily reproducible. Because of the random component of LTMADS, we felt that numerical experiments had to be performed on series of several runs to show the reader the variations in the results.

At each iteration of ORTHOMADS, the main steps for the construction of these directions are as follows. First, the pseudo-random Halton sequence produces one vector in $[0,1]^{n}$ (Subsection 2.1). Second, this vector is scaled and rounded to an appropriate length (Subsection 2.2). The resulting direction is called the adjusted Halton direction. Third, the Householder transformation is then applied to the adjusted Halton direction, producing $n$ orthogonal and integer vectors, forming a basis for $\mathbb{R}^{n}$ (Subsection 2.3). 
Finally, the basis is completed to a positive basis formed by $2 n$ ORTHOMADS POLL directions $D_{k}$, by including in $D_{k}$ the basis and its negatives (Subsection 2.4). Figure 1 summarizes these steps, and will be referred to throughout the section.
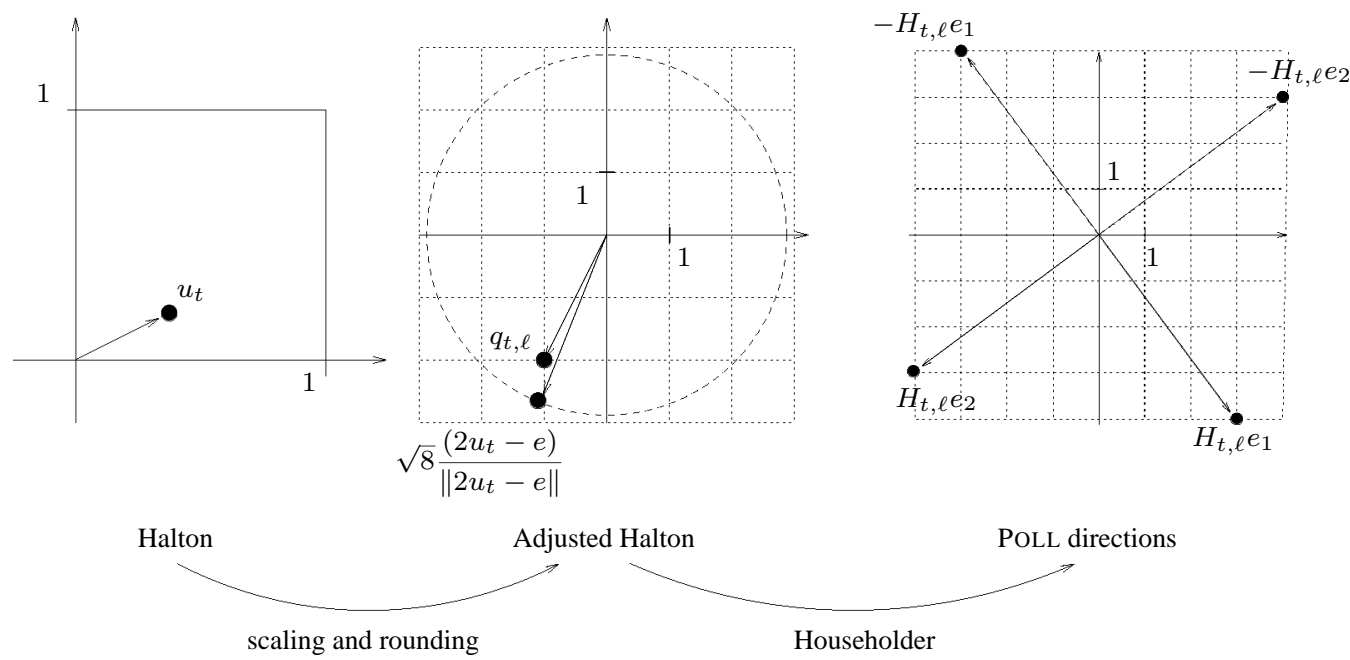

Figure 1: Example with $n=2$ and $(t, \ell)=(6,3)$. The Halton direction is $u_{t}=$ $(3 / 8,2 / 9)^{T}$, the adjusted Halton direction $q_{t, \ell}=(-1,-2)^{T}$ with $\alpha_{t, \ell}=2$ and the set of POLL directions $D_{k}=\left[H_{t, \ell}-H_{t, \ell}\right]$ with $H_{t, \ell} e_{1}=(3,-4)^{T}$ and $H_{t, \ell} e_{2}=(-4,-3)^{T}$. Every POLL direction $d \in D_{k}$ satisfies $\Delta_{k}^{m}\|d\|=5 / 64<\Delta_{k}^{p}=1 / 8$.

In this section we show that the ORTHOMADS directions meet all the conditions detailed in [2,4], so that ORTHOMADS is a valid MADS instance and thus inherits all of its convergence properties.

\subsection{The Halton sequence $u_{t}$}

Halton [11] introduced a deterministic family of sequences that grow dense in the hypercube $[0,1]^{n}$. We consider the simplest sequence of this family, whose $t^{\text {th }}$ element is

$$
u_{t}=\left(u_{t, p_{1}}, u_{t, p_{2}}, \ldots, u_{t, p_{n}}\right)^{T} \in[0,1]^{n}
$$

where $p_{1}=2, p_{2}=3, p_{3}=5$ and $p_{j}$ is the $j^{t h}$ prime number, and $u_{t, p}$ is the radicalinverse function in base $p$. More precisely,

$$
u_{t, p}=\sum_{r=0}^{\infty} \frac{a_{t, r, p}}{p^{1+r}},
$$


where the $a_{t, r, p} \in \mathbb{Z}_{+}$are the unique coefficients of the base $p$ expansion of $t$ :

$$
t=\sum_{r=0}^{\infty} a_{t, r, p} p^{r} .
$$

Table 1 describes the first five elements of $u_{t}$ for $n=4$ (for example, $u_{5,3}=1 \times 3^{-2}+$ $2 \times 3^{-1}=\frac{7}{9}$ ). Our specific sequence of $u_{t}$ vectors is from this point addressed as the sequence of Halton directions.

Table 1: The sequence of Halton directions for $n=4$ and $t=0,1, \ldots, 6$.

\begin{tabular}{|l|rrrr|rrrr|}
\hline$t$ & \multicolumn{5}{|c|}{$t$ in base } & \multicolumn{4}{|c|}{$u_{t}$} \\
& 2 & 3 & 5 & 7 & $u_{t, 2}$ & $u_{t, 3}$ & $u_{t, 5}$ & $u_{t, 7}$ \\
\hline 0 & 0 & 0 & 0 & 0 & 0 & 0 & 0 & 0 \\
1 & 1 & 1 & 1 & 1 & $1 / 2$ & $1 / 3$ & $1 / 5$ & $1 / 7$ \\
2 & 10 & 2 & 2 & 2 & $1 / 4$ & $2 / 3$ & $2 / 5$ & $2 / 7$ \\
3 & 11 & 10 & 3 & 3 & $3 / 4$ & $1 / 9$ & $3 / 5$ & $3 / 7$ \\
4 & 100 & 11 & 4 & 4 & $1 / 8$ & $4 / 9$ & $4 / 5$ & $4 / 7$ \\
5 & 101 & 12 & 10 & 5 & $5 / 8$ & $7 / 9$ & $1 / 25$ & $5 / 7$ \\
6 & 110 & 20 & 11 & 6 & $3 / 8$ & $2 / 9$ & $6 / 25$ & $6 / 7$ \\
\hline
\end{tabular}

In order to remove the linear correlation of the last columns of $u_{t}$, it is proposed in [15] to exclude initial points of the Halton sequence. In the present work, we start the sequence at $t=n+1$.

The following properties will be used in Subsection 2.2:

$$
\begin{aligned}
2 u_{t}-e=0 & \Leftrightarrow n=t=1 \\
\left|2 u_{t, p_{i}}-1\right|=\left|2 u_{t, p_{j}}-1\right| & \Leftrightarrow t=0 .
\end{aligned}
$$

Property (2) follows from the fact that $u_{t, p_{i}}$ and $u_{t, p_{j}}$ can be written as reduced fractions with denominators that are powers of different prime numbers $p_{i}$ and $p_{j}$.

The next result shows that the union of all the directions in the sequence of Halton is dense in $[0,1]^{n}$, i.e. any direction $v \in[0,1]^{n}$ is an accumulation point of the sequence $\left\{u_{t}\right\}_{t=1}^{\infty}$.

Proposition 2.1 The Halton sequence $\left\{u_{t}\right\}_{t=1}^{\infty}$ is dense in $[0,1]^{n}$.

Proof. It suffices to show that for any vector $v \in[0,1]^{n}$ and any $\varepsilon>0$, there exists an integer $t$ such that $\left\|u_{t}-v\right\|<\varepsilon$. A construction of such an integer $t$ involves solving a system of $n$ Diophantine equations, and existence of a solution is ensured by the Chinese Remainder Theorem [8], and by the fact that prime numbers are used in the definition of $u_{t}$. We refer the reader to [11] for a detailed proof. 


\subsection{The adjusted Halton direction $q_{t, \ell}$}

The directions in $D_{k}$ used in the POLL step of MADS cannot be arbitrarily chosen, they must satisfy precise requirements. The Halton directions $u_{t}$ do not satisfy these requirements and the first steps toward generating a satisfactory set $D_{k}$ are to translate, scale and round $u_{t}$.

These operations depend on another integer parameter, $\ell$, which is related to the mesh size parameter $\Delta_{k}^{m}$ (this relationship with $\Delta_{k}^{m}$ is unimportant at this point and will be detailed in Subsection 2.4). The parameter $\ell$ is used to transform the direction $u_{t}$ into the adjusted Halton direction $q_{t, \ell} \in \mathbb{Z}^{n}$, a direction whose norm is close to $2^{|\ell| / 2}$. Furthermore, the normalized direction $\frac{q_{t, \ell}}{\left\|q_{t, \ell}\right\|}$ will be constructed so that it is close to $\frac{2 u_{t}-e}{\left\|2 u_{t}-e\right\|}$. We already observed in (1) that $2 u_{t}-e=0$ is possible only if $n=1$ and $t=1$, and our algorithm never uses $t=1$ (we begin our Halton sequence at $t=n+1$, see Subsection 2.4).

In order to define $q_{t, \ell}$, we first introduce the following sequence of functions:

$$
q_{t}(\alpha)=\operatorname{round}\left(\alpha \frac{2 u_{t}-e}{\left\|2 u_{t}-e\right\|}\right) \in \mathbb{Z}^{n} \cap\left[-\alpha-\frac{1}{2}, \alpha+\frac{1}{2}\right]^{n}
$$

where $\alpha \in \mathbb{R}_{+}$is a scaling factor, and $u_{t}$ is the $t^{t h}$ Halton direction. The function $q_{t}(\cdot)$ is a monotone non-decreasing step function on $\mathbb{R}_{+}$. Let $\alpha_{t, \ell}$ be a scalar such that $\left\|q_{t}\left(\alpha_{t, \ell}\right)\right\|$ is as close as possible to $2^{|\ell| / 2}$, without exceeding it:

$$
\begin{aligned}
& \alpha_{t, \ell} \in \underset{\alpha \in \mathbb{R}_{+}}{\operatorname{argmax}}\left\|q_{t}(\alpha)\right\| \\
& \text { s.t. }\left\|q_{t}(\alpha)\right\| \leq 2^{|\ell| / 2} \text {. }
\end{aligned}
$$

Problem (3) can easily be solved using a bisection method. The adjusted Halton direction $q_{t, \ell}$ is defined to be equal to $q_{t}\left(\alpha_{t, \ell}\right)$, and the following Lemma ensures that $q_{t, \ell}$ is a nonzero integer vector:

Lemma 2.2 If $t \neq 0$, the adjusted Halton direction satisfies $\left\|q_{t, \ell}\right\| \geq 1$.

Proof. From (2), if $t \neq 0$ and $\alpha=\frac{\left\|2 u_{t}-e\right\|}{2\left\|2 u_{t}-e\right\|_{\infty}}$, then $\left\|q_{t}(\alpha)\right\|=1 \leq 2^{|\ell| / 2}$ for all $\ell$.

The following lemma gives a lower bound on the value of $\alpha_{t, \ell}$. It will be used later to justify that $\alpha_{t, \ell}$ grows large with $\ell$.

Lemma 2.3 The optimal solution of Problem (3) satisfies $\alpha_{t, \ell} \geq \frac{2^{|\ell| / 2}}{\sqrt{n}}-\frac{1}{2}$.

Proof. Let $\alpha_{t, \ell}$ be an optimal solution of Problem (3) and set $q_{t, \ell}=q_{t}\left(\alpha_{t, \ell}\right)$. Then every 
feasible solution $\alpha$ to Problem (3) satisfies

$$
\begin{aligned}
\left\|q_{t}(\alpha)\right\|^{2} & =\left\|\operatorname{round}\left(\frac{\alpha\left(2 u_{t}-e\right)}{\left\|2 u_{t}-e\right\|}\right)\right\|^{2} \\
& =\sum_{i=1}^{n} \operatorname{round}\left(\frac{\alpha\left(2 u_{t}^{i}-1\right)}{\left\|2 u_{t}-e\right\|}\right)^{2} \\
& \leq \sum_{i=1}^{n}\left(\alpha+\frac{1}{2}\right)^{2}=n\left(\alpha+\frac{1}{2}\right)^{2} .
\end{aligned}
$$

Define $\beta=\frac{2^{|\ell| / 2}}{\sqrt{n}}-\frac{1}{2}$. Then $\beta$ is feasible for Problem (3), since $\left\|q_{t}(\beta)\right\|^{2} \leq n\left(\beta+\frac{1}{2}\right)^{2}=$ $2^{|\ell|}$; therefore, $\alpha_{t, \ell} \geq \beta$.

Table 2 shows elements of the sequences $u_{t}$ and $q_{t, \ell}$ for $n=4$ and eight pairs $(t, \ell)$ whose values are compatible with the ORTHOMADS algorithm presented in Subsection 2.4. The values of $\alpha_{t, \ell}$ and the square norm $\left\|q_{t, \ell}\right\|^{2}$ are also reported. One can also notice that $\alpha_{t, \ell}$ often differs from $2^{|\ell| / 2}$. In the example illustrated in Figure 1, $(t, \ell)=(6,3)$ and $q_{t}(\alpha)=\operatorname{round}\left(\frac{\alpha}{\sqrt{481}}(-9,-20)^{T}\right)$. An optimal solution of (3) is $\alpha_{t, \ell}=2$ and satisfies $\left\|q_{t, \ell}\right\|=\sqrt{5}<\sqrt{8}=2^{|\ell| / 2}<\left\|q_{t}\left(2^{|\ell| / 2}\right)\right\|=\left\|(-1,-3)^{T}\right\|=\sqrt{10}$.

\begin{tabular}{|c|c|c|c|c|c|c|c|c|c|c|}
\hline \multirow{2}{*}{$(t, \ell)$} & \multicolumn{4}{|c|}{$u_{t}$} & & \multirow{2}{*}{\multicolumn{4}{|c|}{$q_{t, \ell}$}} & \multirow{2}{*}{$\left\|q_{t, \ell}\right\|^{2}$} \\
\hline & $u_{t, 2}$ & $u_{t, 3}$ & $u_{t, 5}$ & $u_{t, 7}$ & & & & & & \\
\hline$(5,0)$ & $5 / 8$ & $7 / 9$ & $1 / 25$ & $5 / 7$ & 1.0 & 0 & 0 & -1 & 0 & 1 \\
\hline$(6,1)$ & $3 / 8$ & $2 / 9$ & $6 / 25$ & $6 / 7$ & 1.0 & 0 & -1 & 0 & 1 & 2 \\
\hline$(7,2)$ & $7 / 8$ & $5 / 9$ & $11 / 25$ & $1 / 49$ & 1.0 & 1 & 0 & 0 & -1 & 2 \\
\hline$(8,3)$ & $1 / 16$ & $8 / 9$ & $16 / 25$ & $8 / 49$ & 2.5 & -2 & 1 & 1 & -1 & 7 \\
\hline$(9,4)$ & $9 / 16$ & $1 / 27$ & $21 / 25$ & $15 / 49$ & 4.0 & 0 & -3 & 2 & -1 & 14 \\
\hline$(10,5)$ & $5 / 16$ & $10 / 27$ & $2 / 25$ & $22 / 49$ & 5.5 & -2 & -1 & -5 & -1 & 31 \\
\hline$(11,6)$ & $13 / 16$ & $19 / 27$ & $7 / 25$ & $29 / 49$ & 7.7 & 5 & 4 & -4 & 2 & 61 \\
\hline$(12,7)$ & $3 / 16$ & $4 / 27$ & $12 / 25$ & $36 / 49$ & 11.0 & -7 & -7 & 0 & 5 & 123 \\
\hline
\end{tabular}

Table 2: The sequence of Halton directions $u_{t}$ and the adjusted Halton directions $q_{t, \ell}$ for $n=4$ and eight pairs $(t, \ell)$.

The following proposition gives a property of the scaling and rounding operations, which transform a vector $v$ into $q=\operatorname{round}(\alpha v /\|u\|)$. The property states that the directions $v /\|v\|$ and $q /\|q\|$ are arbitrarily close for sufficient large values of $\alpha$ : 
Proposition 2.4 Let $v \neq 0$ be a vector in $\mathbb{R}^{n}$. For any $\varepsilon>0$, if $\alpha>\frac{2 \sqrt{n}}{\varepsilon}+\frac{\sqrt{n}}{2}$ and $q=\operatorname{round}\left(\alpha \frac{v}{\|v\|}\right) \neq 0$, then $\left\|\frac{q}{\|q\|}-\frac{v}{\|v\|}\right\|<\frac{\varepsilon}{2}$.

Proof. Consider $\varepsilon>0$ and $\alpha>2 \sqrt{n} / \varepsilon+\sqrt{n} / 2$. The vector $q$ may be expressed as $q=\alpha \frac{v}{\|v\|}+\delta$, where $\delta=\left(\delta_{1}, \delta_{2}, \ldots, \delta_{n}\right)^{T}$ and $\left|\delta_{i}\right|<1 / 2$ for all $i=1,2, \ldots, n$. It follows that

$$
\begin{aligned}
\left\|\frac{q}{\|q\|}-\frac{v}{\|v\|}\right\| & =\left\|\left(\frac{\alpha}{\|q\|}-1\right) \frac{v}{\|v\|}+\frac{\delta}{\|q\|}\right\| \\
& \leq\left\|\left(\frac{\alpha}{\|q\|}-1\right) \frac{v}{\|v\|}\right\|+\left\|\frac{\delta}{\|q\|}\right\| \\
& =\frac{|\alpha-\|q\||}{\|q\|}+\frac{\|\delta\|}{\|q\|} .
\end{aligned}
$$

The norm of $q$ can be bounded with $\alpha \frac{\|v\|}{\|v\|}-\|\delta\| \leq\|q\| \leq \alpha \frac{\|v\|}{\|v\|}+\|\delta\|$ and therefore $|\alpha-\|q\|| \leq\|\delta\|$. Furthermore, $\alpha>2 \sqrt{n} / \varepsilon+\sqrt{n} / 2>\sqrt{n} / 2$ and $\|\delta\|<\sqrt{n} / 2$ implies that $\alpha$ satisfies $0<\alpha-\|\delta\|$. It follows that

$$
\left\|\frac{q}{\|q\|}-\frac{v}{\|v\|}\right\| \leq \frac{2\|\delta\|}{\|q\|} \leq \frac{2\|\delta\|}{\alpha-\|\delta\|}<\frac{\sqrt{n}}{\alpha-\sqrt{n} / 2}<\frac{\varepsilon}{2} .
$$

\subsection{Construction of an orthogonal integer basis}

This subsection gives a way to transform a sequence of directions into a sequence of orthogonal bases. Given an integer nonzero vector $q \in \mathbb{Z}^{n}$, we apply the (symmetric) scaled Householder transformation [12] to construct an orthogonal basis for $\mathbb{R}^{n}$ composed of integer vectors:

$$
H=\|q\|^{2}\left(I_{n}-2 v v^{T}\right), \text { where } v=\frac{q}{\|q\|} .
$$

Proposition 2.5 The columns of $H$ form an integer orthogonal basis for $\mathbb{R}^{n}$.

Proof. First, the columns of $H$ are mutually orthogonal, since $v^{T} v=1$ and

$$
\begin{aligned}
H^{T} H & =\|q\|^{4}\left(I_{n}-2 v v^{T}\right)^{T}\left(I_{n}-2 v v^{T}\right) \\
& =\|q\|^{4}\left(I_{n}-2 v v^{T}-2 v v^{T}+4 v v^{T} v v^{T}\right)=\|q\|^{4} I_{n} .
\end{aligned}
$$


Second, by dividing the previous equation by $\|q\|^{4}$ and applying symmetry, we reveal the inverse of $H$ as $H^{-1}=\frac{1}{\|q\|^{4}} H$. Since $H^{-1}$ exists, the columns of $H$ form a basis in $\mathbb{R}^{n}$. Finally, the entries of

$$
H=\|q\|^{2} I_{n}-2\|q\|^{2} \frac{q}{\|q\|} \frac{q^{T}}{\|q\|}=\|q\|^{2} I_{n}-2 q q^{T}
$$

are integer, since $q$ and $\|q\|^{2}$ are integer.

The next proposition shows that the Householder transformation applied to a dense set of normalized directions produces a dense set of normalized directions:

Proposition 2.6 For $t=1,2, \ldots$, let $v_{t}=\frac{q_{t}}{\left\|q_{t}\right\|}$ and $H_{t}=\left\|q_{t}\right\|^{2}\left(I_{n}-2 v_{t} v_{t}^{T}\right)$. If $\left\{v_{t}\right\}_{t=1}^{\infty}$ is dense on the unit sphere, then the normalized sequence composed of the $i^{\text {th }}$ columns of $H_{t},\left\{\frac{H_{t} e_{i}}{\left\|H_{t} e_{i}\right\|}\right\}_{t=1}^{\infty}$ is dense on the unit sphere.

Proof. Let $w \in \mathbb{R}^{n}$ with $\|w\|=1$ be an arbitrarily unit vector, $\varepsilon>0$ be some small positive number, and $i \in\{1,2, \ldots, n\}$ be the index of a column. For $n>1$ ( $n=1$ is trivial), we need to show that there exists an index $t \in \mathbb{N}$ such that the $i$ th column of $H_{t}$, $H_{t} e_{i}$, satisfies

$$
\left\|\frac{H_{t} e_{i}}{\left\|H_{t} e_{i}\right\|}-w\right\|<\varepsilon .
$$

First, observe that $\left\|H_{t} e_{i}\right\|=\sqrt{e_{i}^{T} H_{t}^{T} H_{t} e_{i}}=\left\|q_{t}\right\|^{2}$, and therefore $\frac{H_{t} e_{i}}{\left\|H_{t} e_{i}\right\|}=e_{i}-2 v_{t} v_{t}^{T} e_{i}$. Now, define the vector

$$
d= \begin{cases}\frac{1}{\sqrt{2\left(1-w_{i}\right)}}\left(e_{i}-w\right) & \text { if } w_{i}<1 \\ e_{i+1}(\text { the sum } i+1 \text { is modulo } n) & \text { otherwise. }\end{cases}
$$

Observe that if $w_{i}=1$ then the vector $d$ satisfies $\|d\|=1$ and $2 d_{i} d=0=e_{i}-w$, and if $w_{i}<1$ then

$$
\begin{aligned}
\|d\| & =\sqrt{d^{T} d}=\sqrt{\frac{1}{2\left(1-w_{i}\right)}\left(e_{i}-w\right)^{T}\left(e_{i}-w\right)}=1 \\
2 d_{i} d & =\frac{1}{\left(1-w_{i}\right)}\left(e_{i}-w\right)_{i}\left(e_{i}-w\right)=e_{i}-w
\end{aligned}
$$

By assumption, $\left\{v_{t}\right\}_{t=1}^{\infty}$ is dense on the unit sphere, and therefore there exists some index $t$ such that $v_{t}=d+\delta$, where $\delta \in \mathbb{R}^{n}$ is small enough to satisfy $\left\|\delta_{i}(d+\delta)+d_{i} \delta\right\|<$ 
$\varepsilon / 2$. The proof may be completed as follows:

$$
\begin{aligned}
\left\|\frac{H_{t} e_{i}}{\left\|H_{t} e_{i}\right\|}-w\right\| & =\left\|e_{i}-2 v_{t} v_{t}^{T} e_{i}-w\right\| \\
& =\left\|e_{i}-2(d+\delta)(d+\delta)^{T} e_{i}-w\right\| \\
& =\left\|e_{i}-2\left(d_{i}+\delta_{i}\right)(d+\delta)-w\right\| \\
& =\left\|e_{i}-2 d_{i} d-w-2\left(\delta_{i}(d+\delta)+d_{i} \delta\right)\right\| \\
& =\left\|e_{i}-\left(e_{i}-w\right)-w-2\left(\delta_{i}(d+\delta)+d_{i} \delta\right)\right\| \\
& =2\left\|\delta_{i}(d+\delta)+d_{i} \delta\right\|<\varepsilon .
\end{aligned}
$$

In Figure 1, the Householder transformation is applied to $q_{t, \ell}=(-1,-2)^{T}$ and produces the integer orthogonal basis $H_{t, \ell}=\left[\begin{array}{rr}3 & -4 \\ -4 & -3\end{array}\right]$.

\subsection{The ORTHOMADS instance of MADS}

The new ORTHOMADS instance of MADS can be now defined by combining the components introduced in Subsections 2.1-2.3. The POLL set $P_{k}$ used by ORTHOMADS at iteration $k$ is entirely determined by the values of the pair $t_{k}$ and $\ell_{k}$. The $t_{k}^{t h}$ element of the Halton sequence $u_{t_{k}}$ is used to create the adjusted Halton direction $q_{t_{k}, \ell_{k}}$ whose norm is as close as possible to $2^{\left|\ell_{k}\right| / 2}$. The Householder transformation on $q_{t_{k}, \ell_{k}}$ produces an orthogonal integer basis $H_{t_{k}, \ell_{k}}$, and the norm of each column is close to $2^{\left|\ell_{k}\right|}$.

The LTMADS and ORTHOMADS algorithms are identical except for the construction of the set $P_{k}$ and the POLL directions $D_{k}$. The set of directions $D=\left[I_{n}-I_{n}\right]$ defining the mesh $M_{k}$ and the mesh update parameters $\tau=4, w^{-}=-1$ and $w^{+}=1$ are the same for both algorithms. The mesh size parameter $\Delta_{k}^{m}$ and the POLL size parameter $\Delta_{k}^{p}$ are still defined with the integer $\ell_{k}$, except that it is allowed to be negative. This extension is not specific to ORTHOMADS and can be applied in LTMADS as well: at each iteration $k$, the POLL and mesh size parameters are entirely defined by the value of $\ell_{k}$ :

$$
\Delta_{k}^{p}=2^{-\ell_{k}} \text { and } \Delta_{k}^{m}=\left\{\begin{array}{cc}
4^{-\ell_{k}} & \text { if } \ell_{k}>0 \\
1 & \text { otherwise. }
\end{array}\right.
$$

At iteration $k=0, \ell_{k}$ is set to 1 and $\Delta_{0}^{m}=\Delta_{0}^{p}=1$. The mesh and POLL size parameters always satisfy $\Delta_{k}^{m} \leq \Delta_{k}^{p}$ and $\Delta_{k}^{m} 2^{\left|\ell_{k}\right|}=\Delta_{k}^{p}$.

In the update step of iteration $k$, if no new incumbent is found, the iteration is said to be unsuccessful and $\ell_{k+1} \leftarrow \ell_{k}+1$. Otherwise, the iteration is a success and $\ell_{k+1} \leftarrow$ $\ell_{k}-1$. The MADS algorithm generates POLL trial points at a distance of order $\Delta_{k}^{p}$ from the POLL center, on a mesh $M_{k}$ of size $\Delta_{k}^{m}$. At an unsuccessful iteration, $\Delta_{k}^{m}$ is reduced 


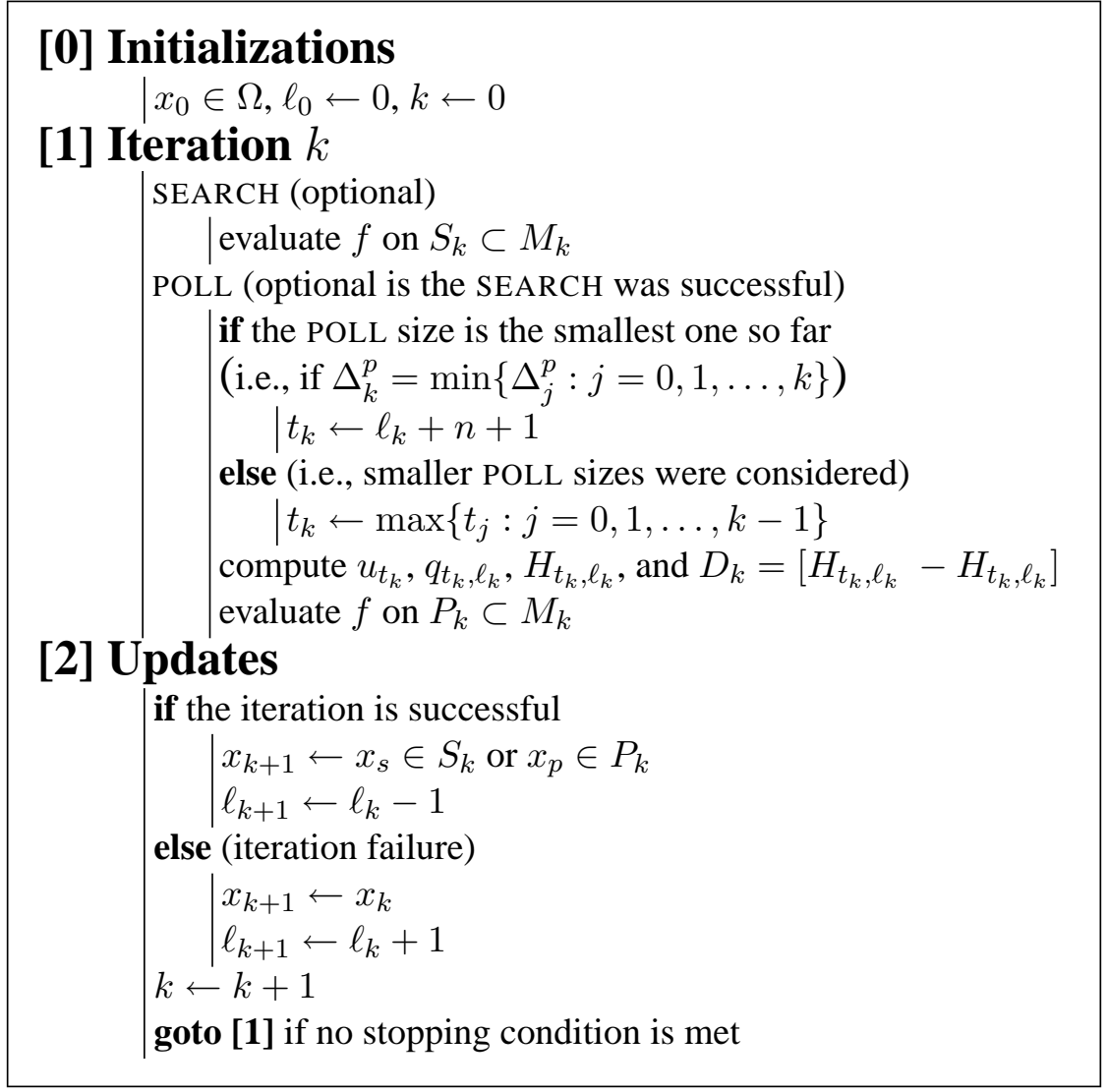

Figure 2: The ORTHOMADS algorithm.

faster than $\Delta_{k}^{p}$ and the number of possible POLL trial points increases, allowing more flexibility in the choice of the POLL directions $D_{k}$.

Figure 2 describes our algorithm. The POLL directions $D_{k}$ depend entirely on the two integers $t_{k}$ and $\ell_{k}$. These integers are chosen to ensure that there will be a sequence of unsuccessful iterations for which the mesh size parameter goes to zero, and such that the directions used in that subsequence will be the tail of the entire Halton sequence. In order to accomplish that goal, we keep track of the value of the smallest POLL size parameter visited so far. At every iteration where $\Delta_{k}^{p}$ is equal to that value, we set $t_{k}=\ell_{k}+n+1$. A consequence of this way of fixing $t_{k}$ is that the set of ordered indices

$$
U:=\left\{k_{1}, k_{2}, \ldots\right\}=\left\{k: \text { iteration } k \text { is unsuccessful, and } \Delta_{k}^{p} \leq \Delta_{j}^{p} \forall j=0,1, \ldots k\right\}
$$

satisfies $\left(t_{k_{1}}, \ell_{k_{1}}\right)=(n+1,0),\left(t_{k_{2}}, \ell_{k_{2}}\right)=(n+2,1), \ldots,\left(t_{k_{i}}, \ell_{k_{i}}\right)=(n+i, i-1)$, and the set of Halton directions $\left\{u_{t_{k}}\right\}_{k \in U}$ is precisely $\left\{u_{t}\right\}_{t=n+1}^{\infty}$.

At the other iterations, those for which smaller POLL sizes were previously considered, we just keep increasing $t_{k}$ so that a new Halton direction is used. Examples of 
Table 3: Example of ORTHOMADS iterations for $n=4$. Iterations $k \in\{4,5,8\}$ correspond to failed iterations with consecutive Halton elements $t_{k}=5,6$ and 7 satisfying $t_{k}=\ell_{k}+n+1$.

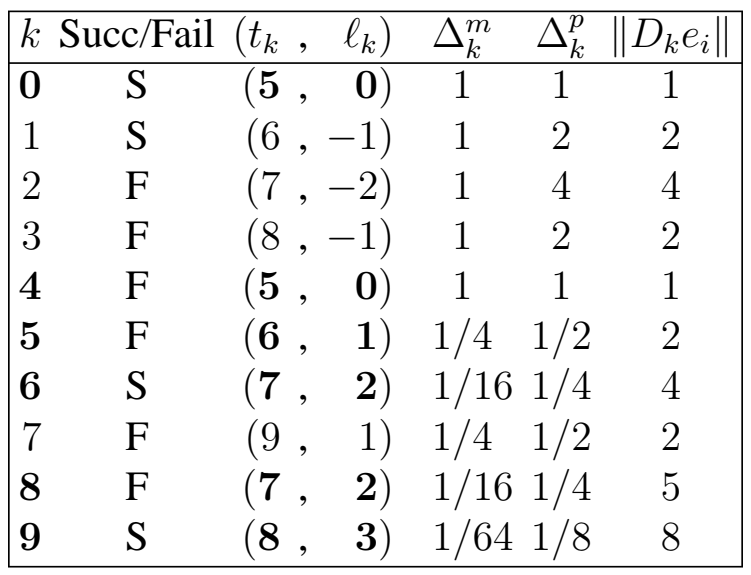

pairs $\left(t_{k}, \ell_{k}\right)$ can be seen in Table 3 . The boldface entries are those where the POLL size parameter is the smallest one so far. In this example, the first three indices of $U$ would be $\{4,5,8\}$.

As in LTMADS, the basis $H_{t_{k}, \ell_{k}}$ is completed to a maximal positive basis composed of $2 n$ directions,

$$
D_{k}=\left[\begin{array}{ll}
H_{t_{k}, \ell_{k}} & -H_{t_{k}, \ell_{k}}
\end{array}\right]
$$

the set of POLL directions. A minimal positive basis with $n+1$ directions is not considered in order to keep orthogonal directions. Table 4 illustrates ORTHOMADS bases $H_{t_{k}, \ell_{k}}$, with possible pairs $\left(t_{k}, \ell_{k}\right)$.

Notice that any direction $D_{k} e_{i}(1 \leq i \leq 2 n)$ satisfies $\left\|D_{k} e_{i}\right\|=\left\|q_{t, \ell}\right\|^{2} \leq\left(2^{|\ell| / 2}\right)^{2}=$ $2^{|\ell|}$ and $\left\|D_{k} e_{i}\right\| \leq 2^{|\ell|}$. Therefore, the POLL trial point $x_{k}+\Delta_{k}^{m} D_{k} e_{i}$ is at an euclidean distance of at most $\Delta_{k}^{m} 2^{|\ell|}=\Delta_{k}^{p}$ from the POLL center. This distance is comparable to that used in LTMADS, where the POLL trial points are exactly at a distance $\Delta_{k}^{p}$ (using the $\ell_{\infty}$ norm) from the POLL center.

We conclude this section with the following propositions that show that ORTHOMADS has the same convergence properties as in [4] with no need for a probabilistic argument.

Proposition 2.7 The set of normalized directions $\left\{\frac{q_{t, \ell}}{\left\|q_{t, \ell}\right\|}\right\}_{t=1}^{\infty}$ with $\ell=t-n-1$ is dense in the unit sphere.

Proof. Let $\varepsilon>0$ and $d \in \mathbb{R}^{n}$ with $\|d\|=1$. Proposition 2.1 states that the Halton sequence $\left\{u_{t}\right\}_{t=1}^{\infty}$ is dense in the unit cube $[0,1]^{n}$. Therefore, there exists an index $t$ such that $\frac{2^{|t-n-1| / 2}}{\sqrt{n}}-\frac{1}{2}>\frac{2 \sqrt{n}}{\varepsilon}+\frac{\sqrt{n}}{2}$ and $\left\|\frac{2 u_{t}-e}{\left\|2 u_{t}-e\right\|}-d\right\| \leq \frac{\varepsilon}{2}$. 
Table 4: A sequence of ORTHOMADS bases corresponding to seven consecutive failed iterations. Pairs $\left(t_{k}, \ell_{k}\right)$ correspond to consecutive Halton elements $t=5,6, \ldots, 12$ with $t_{k}=\ell_{k}+n+1$.

\begin{tabular}{|c|c|c|c|c|c|c|c|c|c|}
\hline $\begin{array}{c}\left(t_{k}, \ell_{k}\right) \\
\left|H_{t_{k}, \ell_{k}} e_{i}\right| \mid\end{array}$ & \multicolumn{4}{|c|}{$H_{t_{k}, \ell_{k}}$} & $\begin{array}{c}\left(t_{k}, \ell_{k}\right) \\
\left\|H_{t_{k}, \ell_{k}} e_{i}\right\|\end{array}$ & \multicolumn{4}{|c|}{$H_{t_{k}, \ell_{k}}$} \\
\hline \multirow[b]{2}{*}{$(5,0)$} & 1 & 0 & 0 & 07 & \multirow[b]{2}{*}{$(9,4)$} & 14 & 0 & 0 & $0^{\circ}$ \\
\hline & 0 & 1 & 0 & 0 & & 0 & -4 & 12 & -6 \\
\hline \multirow[t]{2}{*}{1} & 0 & 0 & -1 & 0 & \multirow[t]{2}{*}{14} & 0 & 12 & 6 & 4 \\
\hline & 0 & 0 & 0 & 1. & & 0 & -6 & 4 & 12 \\
\hline \multirow[b]{2}{*}{$(6,1)$} & 2 & 0 & 0 & $0^{-}$ & \multirow[b]{2}{*}{$(10,5)$} & 23 & -4 & -20 & -4 \\
\hline & 0 & 0 & 0 & 2 & & -4 & 29 & -10 & -2 \\
\hline \multirow[t]{2}{*}{2} & 0 & 0 & 2 & 0 & \multirow[t]{2}{*}{31} & -20 & -10 & -19 & -10 \\
\hline & 0 & 2 & 0 & 0 & & -4 & -2 & -10 & 29 \\
\hline \multirow[b]{2}{*}{$(7,2)$} & 0 & 0 & 0 & 2 & \multirow[b]{2}{*}{$(11,6)$} & 11 & -40 & 40 & -20 \\
\hline & 0 & 2 & 0 & 0 & & -40 & 29 & 32 & -16 \\
\hline \multirow[t]{2}{*}{2} & 0 & 0 & 2 & 0 & \multirow[t]{2}{*}{61} & 40 & 32 & 29 & 16 \\
\hline & 2 & 0 & 0 & 0. & & -20 & -16 & 16 & 53 \\
\hline \multirow[b]{2}{*}{$(8,3)$} & -1 & 4 & 4 & -4 & \multirow[b]{2}{*}{$(12,7)$} & 25 & -98 & 0 & 70 \\
\hline & 4 & 5 & -2 & 2 & & -98 & 25 & 0 & 70 \\
\hline \multirow[t]{2}{*}{7} & 4 & -2 & 5 & 2 & \multirow[t]{2}{*}{123} & 0 & 0 & 123 & 0 \\
\hline & -4 & 2 & 2 & 5. & & 70 & 70 & 0 & 73 \\
\hline
\end{tabular}


Lemma 2.3 ensures that that $\alpha_{t, \ell} \geq \frac{2^{|\ell| / 2}}{\sqrt{n}}-\frac{1}{2}>\frac{2 \sqrt{n}}{\varepsilon}+\frac{\sqrt{n}}{2}$. Combining this last inequality with Proposition 2.4 gives

$$
\begin{aligned}
\left\|\frac{q_{t, \ell}}{\left\|q_{t, \ell}\right\|}-d\right\| & \leq\left\|\frac{q_{t, \ell}}{\left\|q_{t, \ell}\right\|}-\frac{2 u_{t}-e}{\left\|2 u_{t}-e\right\|}\right\|+\left\|\frac{2 u_{t}-e}{\left\|2 u_{t}-e\right\|}-d\right\| \\
& <\frac{\varepsilon}{2}+\frac{\varepsilon}{2}=\varepsilon .
\end{aligned}
$$

This allows us to state our main result:

\section{Theorem 2.8 ORTHOMADS is a valid MADS instance.}

Proof. In order to show that ORTHOMADS is a valid MADS instance we need to show that the POLL directions satisfy the following four properties [2, 4]:

- Any direction $D_{k} e_{i}(1 \leq i \leq 2 n)$ can be written as a non-negative integer combination of the directions of $D$ : This is the case by construction.

- The distance from the POLL center $x_{k}$ to a POLL trial point (in $\ell_{\infty}$ norm) has to be bounded above by $\Delta_{k}^{p}$ : This is also the case by construction because we ensured that $\left\|D_{k} e_{i}\right\| \leq 2^{\left|\ell_{k}\right|}$ for all $i$ in $\{1,2, \ldots, 2 n\}$ and $\left\|\Delta_{k}^{m} D_{k} e_{i}\right\|_{\infty} \leq\left\|\Delta_{k}^{m} D_{k} e_{i}\right\| \leq$ $\Delta_{k}^{m} 2^{\left|\ell_{k}\right|}=\Delta_{k}^{p}$.

- Limits (as defined in [7]) of convergent subsequences of the normalized sets $\overline{D_{k}}=$ $\left\{d /\|d\|: d \in D_{k}\right\}$ are positive spanning sets. This can be shown the same way as in [2] where the proof for LTMADS is detailed, since, for ORTHOMADS and with $\overline{H_{t_{k}, \ell_{k}}}=\left\{d /\|d\|: d \in H_{t_{k}, \ell_{k}}\right\}$, $\operatorname{det}\left(\overline{H_{t_{k}, \ell_{k}}}\right)=-1$.

- The set of normalized directions used over all failed iterations is dense in the unit sphere: The strategy chosen for the values of $t_{k}$ and $\ell_{k}$ ensures that there exists a sequence of failed iterations corresponding to consecutive values of $t_{k}$. These iterations $k \in U$ can be chosen to correspond to large values of $\ell_{k}$ because, from [4], $\lim _{\substack{k \in U \\ k \rightarrow \infty}} \Delta_{k}^{m}=0$, and $\Delta_{k}^{m}=4^{-\ell_{k}}$ for $\ell_{k} \geq 0$. For $k \in U$, the sets of directions $\left\{D_{k}\right\}_{k \in U}$ are constructed from consecutive directions $q_{t_{k}, \ell_{k}}$, which are dense in the unit sphere after normalization (Proposition 2.7). Then, from Proposition 2.6 and since $D_{k}=\left[H_{t_{k}, \ell_{k}}-H_{t_{k}, \ell_{k}}\right]$, the set of normalized directions $\left\{\frac{D_{k} e_{i}}{\left\|D_{k} e_{i}\right\|}\right\}_{k \in U}$ is also dense in the unit sphere for all $i=1,2, \ldots, 2 n$. 


\section{Numerical Tests}

In this section, ORTHOMADS is compared to its predecessor LTMADS [4] and to the GPS method [18], on 45 problems from the literature. In the MADS algorithms, the theory supports handling constraints by the extreme barrier approach: Points outside $\Omega$ are simply ignored and $f$ is not evaluated. For GPS, the extreme barrier approach is supported by the theory only for a finite number of linear constraints [13]. Still, for comparison, we apply two different approaches: the extreme barrier (GPS-EB), and the filter method described in [3] (GPS-FILTER), which has stronger theoretical support.

Because of its random behavior, 30 instances of LTMADS are performed for each problem. GPS and ORTHOMADS are scored by comparing them against the 30 LTMADS instances. A score of $s$ for GPS or ORTHOMADS means that this instance gave a value of $f$ at least as good as $s$ of the 30 LTMADS instances, with a relative precision of $1 \%$. The worst score is 0 and the perfect score corresponds to 30 . We consider that a bad instance has a score less than 10, an acceptable instance is between 10 and 19, and a good instance has a score greater than or equal to 20 .

The integer $\ell_{k}$ (see (5)), defining the mesh and POLL size parameters $\Delta_{k}^{m}$ and $\Delta_{k}^{p}$ at iteration $k$, is allowed to be negative for both LTMADS and ORTHOMADS. Maximal positive bases ( $2 n$ directions) are used in the three methods, as is the opportunistic strategy (the POLL is interrupted at the first success), and the optimist strategy: after a successful point has been found, a SEARCH point is generated further along the same direction. No other SEARCH is performed. The stopping criteria is satisfied when the POLL size parameter $\Delta_{k}^{p}$ drops below $1 \mathrm{E}-12$ or when the number of function evaluations reaches $1000 n$.

The methods are tested on 45 problems divided into 4 groups: our choice of smooth and nonsmooth unconstrained problems is the same as in [10] and [9], respectively, with 21 smooth problems from the CUTEr test set [16] and 13 nonsmooth problems from [14], which is a compilation of nonsmooth problems from the literature. We also tested on 9 constrained problems from [5, 6, 14], and in addition, we added two problems from [1] that correspond to real applications.

All results and problem descriptions are summarized in Tables 5-9, where $f\left(x^{*}\right)$ corresponds to the best known minimal value of $f$, value to the final value of $f$ for each method, and evals to the number of function evaluations that each method performed. Tables 5 and 6 show results on the 21 unconstrained smooth problems from CUTEr. ORTHOMADS has a perfect score on 17 of these problems. Table 7 displays results on the 13 unconstrained nonsmooth problems, where ORTHOMADS achieves good scores

on 7 problems. Table 8 shows results for the 9 constrained problems. The same number of problems (4) is considered good and bad for ORTHOMADS. Finally, Table 9 presents results for the two real applications, and ORTHOMADS has perfect scores on both of them.

Table 10 summarizes the results. The first observation is that both MADS instances 
Table 5: CuTEr unconstrained smooth problems (1 of 2). A score of $s$ for a method indicates that the final $f$ value is at least as good as $s$ of the 30 LTMADS runs (with a relative error of $1 \%$ ).

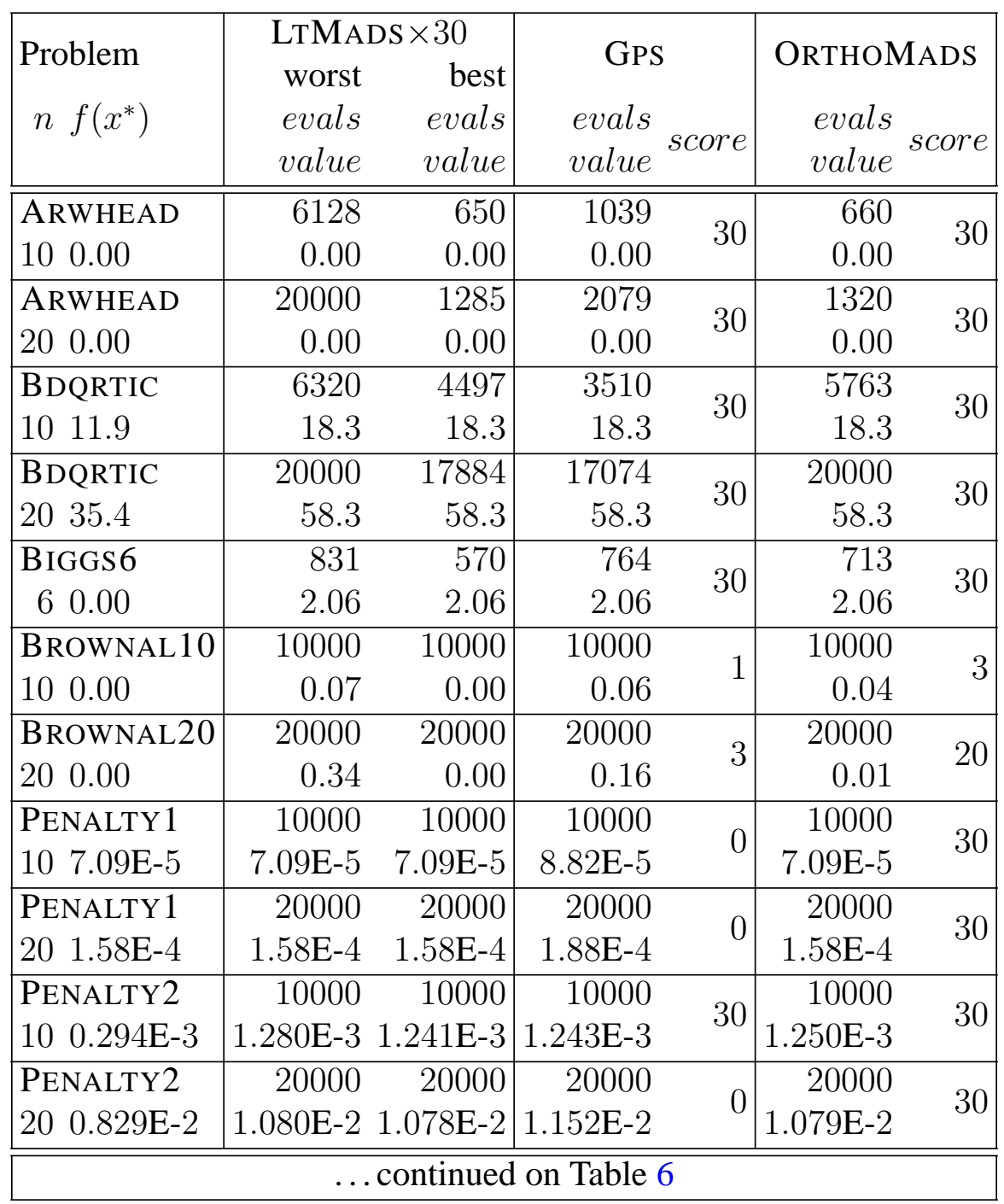


Table 6: CUTEr unconstrained smooth problems (2 of 2). A score of $s$ for a method indicates that the final $f$ value is at least as good as $s$ of the 30 LTMADS runs (with a relative error of $1 \%$ ).

\begin{tabular}{|c|c|c|c|}
\hline $\begin{array}{l}\text { Problem } \\
n f\left(x^{*}\right)\end{array}$ & $\begin{array}{c}\text { LTMADS } \times 30 \\
\text { worst } \\
\text { evals } \\
\text { vevalue } \\
\text { value value }\end{array}$ & $\begin{array}{c}\text { GPS } \\
\text { evals } \\
\text { value }\end{array}$ & $\begin{array}{l}\text { ORTHOMADS } \\
\text { evals } \\
\text { value score }\end{array}$ \\
\hline $\begin{array}{l}\text { POWELLSG } \\
120.00\end{array}$ & $\begin{array}{rr}12000 & 12000 \\
0.00 & 0.00\end{array}$ & $\begin{array}{r}10093 \\
0.00\end{array}$ & $\begin{array}{r}12000 \\
0.00\end{array}$ \\
\hline $\begin{array}{l}\text { POWELLSG } \\
200.00\end{array}$ & $\begin{array}{rr}20000 & 20000 \\
0.00 & 0.00\end{array}$ & $\begin{array}{r}20000 \\
0.00\end{array}$ & $\begin{array}{r}20000 \\
0.00\end{array}$ \\
\hline $\begin{array}{l}\text { SROSENBR } \\
100.00\end{array}$ & $\begin{array}{rr}10000 & 10000 \\
6.31 & 0.00\end{array}$ & $\begin{array}{r}10000 \\
0.00\end{array}$ & $\begin{array}{r}10000 \\
0.06\end{array}$ \\
\hline $\begin{array}{l}\text { SROSENBR } \\
200.00\end{array}$ & $\begin{array}{rr}20000 & 20000 \\
16.52 & 0.48\end{array}$ & $\begin{array}{r}20000 \\
0.00\end{array}$ & $\begin{array}{r}1958 \\
0.00\end{array}$ \\
\hline $\begin{array}{l}\text { TRIDIA } \\
100.00\end{array}$ & $\begin{array}{rr}10000 & 7591 \\
0.00 & 0.00\end{array}$ & $\begin{array}{r}9317 \\
0.00\end{array}$ & $\begin{array}{r}10000 \\
0.00\end{array}$ \\
\hline $\begin{array}{l}\text { TRIDIA } \\
200.00\end{array}$ & $\begin{array}{rr}20000 & 20000 \\
0.00 & 0.00\end{array}$ & $\begin{array}{r}20000 \\
0.00\end{array}$ & $\begin{array}{r}20000 \\
0.00\end{array}$ \\
\hline $\begin{array}{l}\text { VARDIM } \\
100.00\end{array}$ & $\begin{array}{r}1000 \\
0.00\end{array}$ & $\begin{array}{r}10000 \\
4.01\end{array}$ & $\begin{array}{r}10000 \\
0.00\end{array}$ \\
\hline $\begin{array}{l}\text { VARDIM } \\
200.00\end{array}$ & $\begin{array}{rr}20000 & 20000 \\
0.00 & 0.00\end{array}$ & $\begin{array}{r}20000 \\
110.59\end{array}$ & $\begin{array}{r}20000 \\
110.84\end{array}$ \\
\hline $\begin{array}{l}\text { WoODS } \\
120.00\end{array}$ & $\begin{array}{rr}10951 & 7327 \\
104.91 & 104.91\end{array}$ & $\begin{array}{r}8433 \\
104.91\end{array}$ & $\begin{array}{r}9479 \\
104.91\end{array}$ \\
\hline $\begin{array}{l}\text { WoODS } \\
200.00\end{array}$ & $\begin{array}{rr}20000 & 19181 \\
174.84 & 174.84\end{array}$ & \begin{tabular}{|r}
20000 \\
174.84
\end{tabular} & $\begin{array}{r}20000 \\
174.84\end{array}$ \\
\hline \multicolumn{3}{|c|}{ "average scores } & 26.6 \\
\hline
\end{tabular}


Table 7: Results for unconstrained nonsmooth problems from [14]. A score of $s$ for a method indicates that the final $f$ value is at least as good as $s$ of the 30 LTMADS runs (with a relative error of $1 \%$ ).

\begin{tabular}{|c|c|c|c|c|c|c|}
\hline $\begin{array}{l}\text { Problem } \\
\qquad n f\left(x^{*}\right)\end{array}$ & $\begin{array}{c}\text { LTMAI } \\
\text { worst } \\
\text { evals } \\
\text { value }\end{array}$ & $\begin{array}{r}\mathrm{DS} \times 30 \\
\text { best } \\
\text { evals } \\
\text { value }\end{array}$ & $\begin{array}{l}\text { GPS } \\
\text { evals } \\
\text { value }\end{array}$ & score & $\begin{array}{c}\text { ORTHON } \\
\text { evals } \\
\text { value }\end{array}$ & score \\
\hline ELATTAR & 456 & 1795 & 2392 & & 3984 & \\
\hline 60.560 & 8.021 & 0.563 & 1.714 & 16 & 1.504 & 20 \\
\hline EvD61 & 490 & 3280 & 920 & & 4224 & \\
\hline 60.0349 & 1.6001 & 0.0417 & 0.5443 & 5 & 0.0709 & $2 Z$ \\
\hline FILTER & 1293 & 1761 & 1132 & 7 & 1332 & 0 \\
\hline 90.00619 & 0.00971 & 0.00797 & 0.00950 & $\gamma$ & 0.00935 & $y$ \\
\hline GOFFIN & 50000 & 50000 & 24097 & & 16842 & \\
\hline $50 \quad 0.00$ & 1.10 & 0.06 & 0.00 & 30 & 0.00 & 30 \\
\hline Hs78 & 403 & 1026 & 819 & & 405 & \\
\hline $5-2.92$ & 10.00 & -2.88 & 0.00 & 13 & 0.00 & 13 \\
\hline L1HILB & 17953 & 50000 & 8738 & 0 & 50000 & 14 \\
\hline 500.00 & 1.84 & 0.04 & 3.95 & 0 & 0.22 & 14 \\
\hline MXHILB & 11523 & 20377 & 9384 & 0 & 20755 & 3 \\
\hline 500.00 & 0.280 & 0.003 & 0.976 & 0 & 0.197 & 3 \\
\hline OSBORNE2 & 2046 & 5414 & 1660 & & 4555 & ח \\
\hline 110.0480 & 0.1703 & 0.0549 & 0.2799 & U & 0.1089 & 20 \\
\hline PBC1 & 1211 & 1127 & 677 & $?$ & 1291 & 17 \\
\hline 50.0223 & 0.4146 & 0.0343 & 0.3845 & 2 & 0.1602 & 17 \\
\hline POLAK2 & 1449 & 1742 & 1327 & 30 & 949 & 30 \\
\hline 1054.6 & 54.6 & 54.6 & 54.6 & & 54.6 & 30 \\
\hline SHOR & 1345 & 882 & 787 & 0 & 1087 & 30 \\
\hline 522.6 & 22.9 & 22.6 & 23.5 & U & 22.8 & 30 \\
\hline WONG1 & 1161 & 2109 & 1100 & 30 & 1823 & 30 \\
\hline 7681 & 699 & 693 & 697 & 30 & 693 & 30 \\
\hline WONG2 & 5403 & 5403 & 1871 & & 4977 & \\
\hline 1024.3 & 31.4 & 24.8 & 47.4 & 0 & 32.8 & 0 \\
\hline \multicolumn{4}{|c|}{ "average scores } & 10.2 & & 18.3 \\
\hline
\end{tabular}


Table 8: Results for constrained problems. A score of $s$ for a method indicates that the final $f$ value is at least as good as $s$ of the 30 LTMADS runs (with a relative error of $1 \%$ ).

\begin{tabular}{|c|c|c|c|c|c|c|}
\hline $\begin{array}{l}\text { Problem } \\
\qquad n m f\left(x^{*}\right)\end{array}$ & $\begin{array}{c}\text { LTMAI } \\
\text { worst } \\
\text { evals } \\
\text { value }\end{array}$ & $\begin{array}{r}\mathrm{S} \times 30 \\
\text { best } \\
\text { evals } \\
\text { value }\end{array}$ & $\begin{array}{l}\text { GPS-FILTER } \\
\text { evals } \\
\text { value }\end{array}$ & $\begin{array}{l}\text { GPS-E } \\
\text { evals } \\
\text { value }\end{array}$ & & $\begin{array}{l}\text { ORTHOMADS } \\
\text { evals } \\
\text { value }\end{array}$ \\
\hline CRESCENT 10 [5] & 121279 & 4473 & 2152 & 1172 & & 5497 \\
\hline $\begin{array}{ll}10 \quad 2-9.00\end{array}$ & -8.26 & -8.95 & -6.19 & -2.32 & 0 & -8.97 \\
\hline DISK10 [5] & 1909 & 2626 & 2322 & 1143 & & 2359 \\
\hline $10 \quad 1-17.3$ & -17.2 & -17.3 & -13.0 & -10.0 & 0 & -17.2 \\
\hline B250 [6] & 60000 & 60000 & 15412 & 27773 & & 60000 \\
\hline $\begin{array}{lll}60 & 1 & 7.95\end{array}$ & 15.41 & 7.99 & 1142.01 & 1116.03 & 0 & 16.92 \\
\hline B500 [6] & 16705 & 15359 & 11189 & 18912 & & 29858 \\
\hline $\begin{array}{lll}60 & 1 & 104\end{array}$ & 557 & 104 & 1235 & 1254 & 0 & 277 \\
\hline G2 [6] & 3880 & 6461 & 2056 & 2689 & & 5414 \\
\hline $10 \quad 2-0.728$ & -0.181 & -0.728 & -0.221 & -0.706 & 29 & -0.561 \\
\hline G2 [6] & 10877 & 15722 & 6376 & 6551 & & 20000 \\
\hline $20 \quad 2-0.804$ & -0.203 & -0.736 & -0.241 & -0.721 & 2 & -0.711 \\
\hline Hs $114[14]$ & 1506 & 2135 & 1756 & 1756 & & 1661 \\
\hline $9 \quad 6-1769$ & -1012 & -1312 & -968 & -968 & 0 & -1016 \\
\hline MAD6 [14] & 1122 & 1542 & 1378 & 1378 & & 1671 \\
\hline $\begin{array}{lll}5 & 7 & 0.102\end{array}$ & 0.113 & 0.102 & 0.103 & 0.103 & 22 & 0.108 \\
\hline PENTAGON [14] & 859 & 2525 & 601 & 601 & & 980 \\
\hline $615-1.86$ & -1.60 & -1.86 & 0.00 & 0.00 & 0 & -1.81 \\
\hline \multicolumn{4}{|c|}{ " average scores } & & 8.9 & 16.3 \\
\hline
\end{tabular}


Table 9: Results for real applications. A score of $s$ for a method indicates that the final $f$ value is at least as good as $s$ of the 30 LTMADS runs (with a relative error of 1\%). Displayed $z$ values for problem STY are divided by $10^{7}$.

\begin{tabular}{|c|c|c|c|c|}
\hline $\begin{array}{l}\text { Problem } \\
\qquad n m f\left(x^{*}\right)\end{array}$ & \begin{tabular}{cr}
\multicolumn{2}{|c|}{ LTMADS $\times 30$} \\
worst & best \\
evals & evals \\
value & value
\end{tabular} & $\begin{array}{l}\text { GPS-FILTER } \\
\text { evals } \\
\text { value }\end{array}$ & $\begin{array}{l}\text { GPS-EB } \\
\text { evals } \\
\text { value }\end{array}$ & $\begin{array}{l}\text { ORTHOMADS } \\
\text { evals } \\
\text { value score }\end{array}$ \\
\hline $\begin{array}{l}\text { MDO [1] } \\
1010-3964\end{array}$ & $\begin{array}{rr}1767 & 15 \\
-2530 & -3964\end{array}$ & $\begin{array}{r}2719 \\
-1386\end{array}$ & $\begin{array}{r}2048 \\
-1386\end{array}$ & $\begin{array}{r}1212 \\
-3964\end{array}$ \\
\hline $\begin{array}{l}\mathrm{STY}[1] \\
811-3.35\end{array}$ & $\begin{array}{|rr|}1590 & 1189 \\
-2.88 & -3.29 \\
\end{array}$ & $\begin{array}{r}2073 \\
-3.11 \\
\end{array}$ & $\begin{array}{r}2113 \\
-2.82 \\
\end{array}$ & $\begin{array}{r}1214 \\
-3.27 \\
\end{array}$ \\
\hline \multicolumn{3}{|c|}{$\begin{array}{ll}\text { average scores } \\
\end{array}$} & 0.0 & 30.0 \\
\hline
\end{tabular}

outperform GPS. For 25 problems out of 45, ORTHOMADS found the same solution as the best of 30 LTMADS runs. The new method solved 32 problems out of 45 problems efficiently enough that, for these problems, the single run of ORTHOMADS was better than two thirds of the 30 LTMADS runs. For 4 problems, the two methods performed equally well, and for 9 problems, at least two thirds of the LTMADS runs gave a better solution than the one produced by ORTHOMADS.

Table 10: Summary for the GPS and ORTHOMADS performances. F, E and O correspond respectively to GPS-FILTER, GPS-EB, and ORThOMADS. A bad instance has a score between 0 and 9, an acceptable (acc.) instance a score between 10 and 19, a good instance a score higher than 20 and a perfect (perf.) instance has a score of 30.

\begin{tabular}{|r|cc|c|c|ccc|ccc|ccc|ccc|}
\hline \multirow{2}{*}{ problems } & \multicolumn{2}{|c|}{$\begin{array}{c}\text { average } \\
\text { scores (on 30) }\end{array}$} & $\begin{array}{c}\text { \# of } \\
\text { problems }\end{array}$ & $\begin{array}{c}\text { \# of bad } \\
\text { instances }\end{array}$ & $\begin{array}{c}\text { \# of acc. } \\
\text { instances }\end{array}$ & $\begin{array}{c}\text { \# of good } \\
\text { instances }\end{array}$ & $\begin{array}{c}\text { \# of perf. } \\
\text { instances }\end{array}$ \\
\hline \hline & $\mathrm{F}$ & $\mathrm{E}$ & $\mathrm{O}$ & & $\mathrm{F}$ & $\mathrm{E}$ & $\mathrm{O}$ & $\mathrm{F}$ & $\mathrm{E}$ & $\mathrm{O}$ & $\mathrm{F}$ & $\mathrm{E}$ & $\mathrm{O}$ & $\mathrm{F}$ & $\mathrm{E}$ & $\mathrm{O}$ \\
\hline smooth & 20.2 & 20.2 & 26.6 & 21 & 7 & 7 & 2 & 0 & 0 & 0 & 14 & 14 & 19 & 14 & 14 & 17 \\
nonsmooth & 10.2 & 10.2 & 18.3 & 13 & 8 & 8 & 3 & 2 & 2 & 3 & 3 & 3 & 7 & 3 & 3 & 4 \\
constrained & 3.2 & 8.9 & 16.3 & 9 & 8 & 6 & 4 & 0 & 0 & 1 & 1 & 3 & 4 & 0 & 0 & 2 \\
real appli. & 11.0 & 0.0 & 30.0 & 2 & 1 & 2 & 0 & 0 & 0 & 0 & 1 & 0 & 2 & 0 & 0 & 2 \\
\hline \hline total or avg & 13.5 & 14.2 & 22.3 & 45 & 24 & 23 & 9 & 2 & 2 & 4 & 19 & 20 & 32 & 17 & 17 & 25 \\
\hline
\end{tabular}


Figure 3 illustrates the spread of the directions for both LTMADS and ORTHOMADS. Rosenbrock's function [17] with $n=2$ and $n=3$ was used with 2000 and 3000 evaluations, respectively. In the two-dimensional case, all the normalized directions used to generate POLL trial points are directly represented on the top two subfigures. It is clear that ORTHOMADS directions are well distributed on the unit circle. This is not the case with LTMADS because half the directions correspond to either $\pm e_{1}$ or $\pm e_{2}$. For $n=3$, the two plots on the bottom represent the standard angles of the normalized directions in spherical coordinates. There again it can be seen that ORTHOMADS directions have a better distribution than those of LTMADS, since at least two thirds of the LTMADS directions possess some null coordinates. On the subfigure using LTMADS with $n=3$, the horizontal bar at $\Phi=\pi / 2$ corresponds to the set of directions where $z=0$. The vertical bars at $\theta= \pm \pi / 2$ correspond to directions with $x=0$, and the one at $\theta=0$ and $\theta=\pi$ correspond to directions with $y=0$.
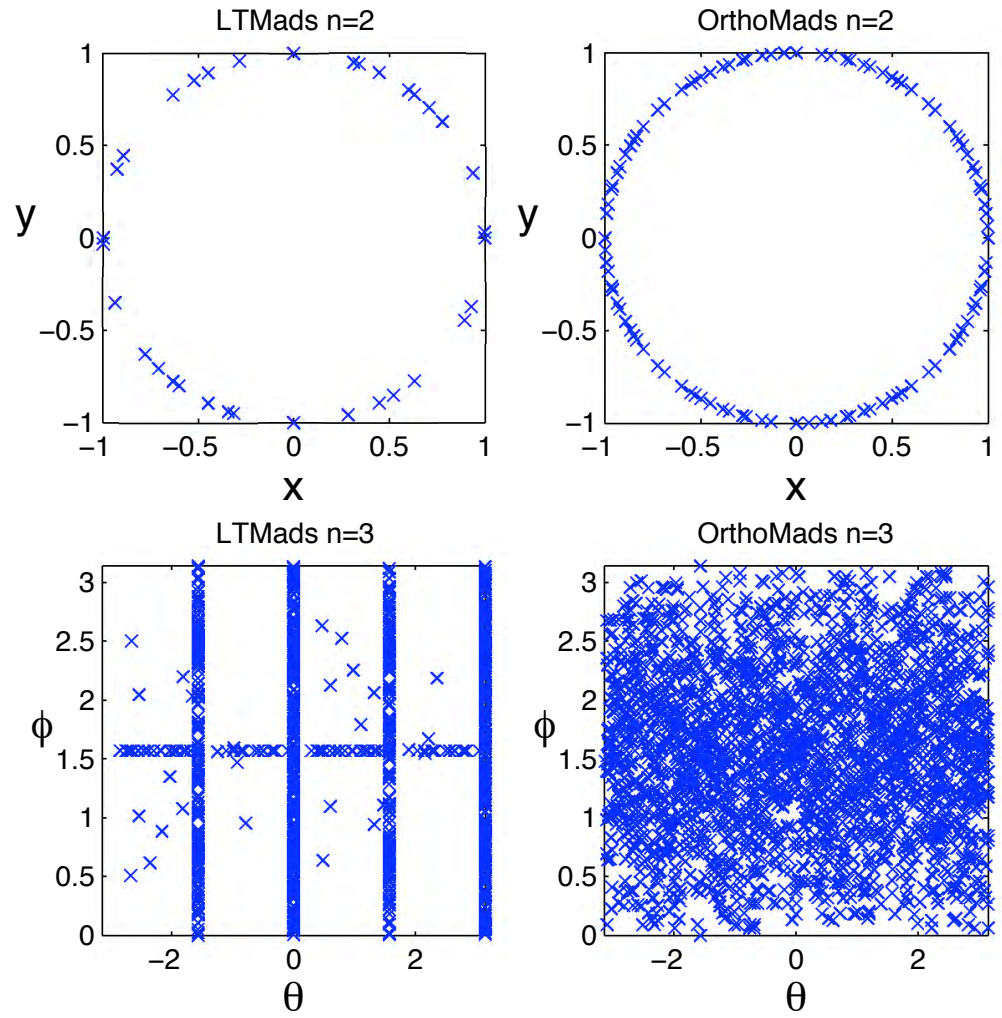

Figure 3: LTMADS and ORTHOMADS normalized POLL directions on the Rosenbrock function with $n=2$ and $n=3$. 


\section{Discussion}

This paper introduced ORTHOMADS, an alternative instantiation of the MADS class of algorithms. The advantages of ORTHOMADS over the original LTMADS are that the MADS directions are chosen deterministically, and that those directions are orthogonal to each other. Moreover, ORTHOMADS inherits all of the MADS convergence properties, without probabilistic arguments, and without additional parameters.

Intensive tests on 45 problems from the literature showed that both MADS instances outperform the GPS algorithm, and that ORTHOMADS is at least as competitive as LTMADS, with a better distribution of the POLL directions.

\section{Acknowledgements}

We would like to thank Ana Custódio and Luis Vicente for helpful remarks about missed cones of directions in single iterations of LTMADS. These remarks and similar observations of our own helped motivate us to discover ORTHOMADS. We also wish to thank Andrew Booker for suggesting quasi-Monte Carlo methods which started us in directions that led to our use of Halton sequences.

\section{References}

[1] C. Audet, V. Béchard, and S. Le Digabel. Nonsmooth optimization through Mesh Adaptive Direct Search and Variable Neighborhood Search. To appear in Journal of Global Optimization, DoI: 10.1007/s10898-007-9234-1, 2007.

[2] C. Audet, A. L. Custódio, and J. E. Dennis, Jr. Erratum : Mesh Adaptive Direct Search Algorithms for Constrained Optimization. SIAM Journal on Optimization, 18(4):15011503, 2008.

[3] C. Audet and J. E. Dennis, Jr. A Pattern Search Filter Method for Nonlinear Programming without Derivatives. SIAM Journal on Optimization, 14(4):980-1010, 2004.

[4] C. Audet and J. E. Dennis, Jr. Mesh Adaptive Direct Search Algorithms for Constrained Optimization. SiAM Journal on Optimization, 17(1):188-217, 2006.

[5] C. Audet and J. E. Dennis, Jr. A Mads Algorithm with a Progressive Barrier for DerivativeFree Nonlinear Programming. Technical Report G-2007-37, Les Cahiers du GERAD, May 2007.

[6] C. Audet, J. E. Dennis, Jr., and S. Le Digabel. Parallel Space Decomposition of the Mesh Adaptive Direct Search Algorithm. Technical Report G-2007-81, Les Cahiers du GERAD, November 2007. 
[7] I. D. Coope and C. J. Price. Frame-Based Methods for Unconstrained Optimization. Journal of Optimization Theory and Applications, 107(2):261-274, 2000.

[8] T. H. Cormen, C. E. Leiserson, R. L. Rivest, and C. Stein. Introduction to Algorithms, Second Edition, pages 873-876. Mit Press and McGraw-Hill, 2001.

[9] A. L. Custódio, J. E. Dennis, Jr., and L. N. Vicente. Using Simplex Gradients of Nonsmooth Functions in Direct Search Methods. To appear in IMA Journal of Numerical Analysis, 2007.

[10] A. L. Custódio and L. N. Vicente. Using Sampling and Simplex Derivatives in Pattern Search Methods. SiAm Journal on Optimization, 18:537-555, 2007.

[11] J. H. Halton. On the Efficiency of Certain Quasi-Random Sequences of Points in Evaluating Multi-Dimensional Integrals. Numerische Mathematik, 2(1):84-90, 1960.

[12] A. S. Householder. Unitary Triangularization of a Nonsymmetric Matrix. Journal of the Association for Computing Machinery (ACM), 5(4):339-342, 1958.

[13] R. M. Lewis and V. Torczon. Pattern Search Methods for Linearly Constrained Minimization. SiAm Journal on Optimization, 10(3):917-941, 2000.

[14] L. Lukšan and J. Vlček. Test Problems for Nonsmooth Unconstrained and Linearly Constrained Optimization. Technical Report V-798, ICS As CR, February 2000.

[15] J. Matoušek. On the $\mathrm{L}_{2}$-Discrepancy for Anchored Boxes. Journal of Complexity, 14(4):527-556, 1998.

[16] D. Orban N. I. M. Gould and Ph. L. Toint. Cuter (and SifDeC): a Constrained and Unconstrained Testing Environment, revisited. ACM Transactions on Mathematical Software, 29(4):373-394, 2003.

[17] H. H. Rosenbrock. An Automatic Method for Finding the Greatest or Least Value of a Function. The Computer Journal, 3(3):175-184, October 1960.

[18] V. Torczon. On the Convergence of Pattern Search Algorithms. Siam Journal on Optimization, 7(1):1-25, February 1997. 